\title{
Probing Allostery Through DNA
}

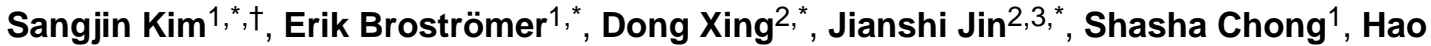 \\ $\mathrm{Ge}^{2,4}$, Siyuan Wang ${ }^{1}$, Chan $\mathrm{Gu}^{5}$, Lijiang Yang ${ }^{5}$, Yi Qin Gao ${ }^{5}$, Xiao-dong Su${ }^{2, \S}$, Yujie Sun ${ }^{2, \S}$, \\ and X. Sunney $\mathbf{X i e}^{1,2, \S}$ \\ ${ }^{1}$ Department of Chemistry and Chemical Biology, Harvard University, Cambridge, MA 02138, \\ USA
}

${ }^{2}$ Biodynamic Optical Imaging Center (BIOPIC), School of Life Sciences, Peking University, Beijing 100871, China

${ }^{3}$ Academy for Advanced Interdisciplinary Studies, Peking University, Beijing 100871, China

${ }^{4}$ Beijing International Center for Mathematical Research, Peking University, Beijing 100871, China

${ }^{5}$ Institute of Theoretical and Computational Chemistry, College of Chemistry and Molecular Engineering, Peking University, Beijing 100871, China

\section{Abstract}

\begin{abstract}
Allostery is well documented for proteins but less recognized for DNA-protein interactions. Here we report that specific binding of a protein on DNA is substantially stabilized or destabilized by another protein bound nearby. The ternary complex's free energy oscillates as a function of the separation between the two proteins with a periodicity of $\sim 10$ base pairs, the helical pitch of Bform DNA, and a decay length of $\sim 15$ base pairs. The binding affinity of a protein near a DNA hairpin is similarly dependent on their separation, which - together with molecular dynamics simulations-suggests that deformation of the double-helical structure is the origin of DNA allostery. The physiological relevance of this phenomenon is illustrated by its effect on gene expression in live bacteria and on a transcription factor's affinity near nucleosomes.
\end{abstract}

Upon binding of a ligand, a macromolecule often undergoes conformational changes that modify the binding affinity of a second ligand at a distant site. This phenomenon, known as "allostery", is responsible for dynamic regulation of biological functions. Although extensive studies have been done on allostery in proteins or enzymes $(1,2)$, less is known for that through DNA, which is normally considered as a mere template providing binding sites. In fact, multiple proteins, such as transcription factors and RNA or DNA polymerases, bind close to each other on genomic DNA to carry out their cellular functions in concert. Such allostery through DNA has been implicated in previous studies (3-10) but has not been quantitatively characterized or mechanistically understood.

We performed a single-molecule study of allostery through DNA by measuring the dissociation rate constant $\left(k_{o f f}\right)$ of a DNA-bound protein affected by the binding of another protein nearby. In the assay, DNA duplexes (dsDNA), tethered on the passivated surface of a flow cell, contained two specific protein binding sites separated by a linker sequence of $L$ base pairs (bp) (Fig. 1A and figs. S1 and S2) (11). One of the proteins was fluorescently

\footnotetext{
$\S_{\text {}}$ To whom correspondence should be addressed. xdsu@pku.edu.cn (X.-D.S.); sun_yujie@pku.edu.cn (Y.S.); xie @ chemistry.harvard.edu (X.S.X.).

${ }^{*}$ These authors contributed equally to this work.

${ }^{\dagger}$ Present address: Department of Molecular Cellular and Developmental Biology, Yale University, New Haven, CT 06511, USA.
} 
labeled, and many individual protein-DNA complexes were monitored in a large field of view with a total internal reflection fluorescence microscope. Once the labeled protein molecules were bound to DNA, the second protein at a certain concentration was flowed in. Stochastic dissociation times of hundreds of labeled protein molecules were then recorded, the average of which yields the $k_{\text {off }}$ (fig. S3) (12).

We first present a protein pair that does not substantially bend DNA, namely a Cy3B-labeled DNA-binding domain of glucocorticoid receptor (GRDBD), a eukaryotic transcription factor, together with BamHI, a type II endonuclease (Fig. 1A) (13, 14). To prevent the endonuclease activity of BamHI, we used buffer containing $\mathrm{Ca}^{2+}$ instead of $\mathrm{Mg}^{2+}$. At a saturating concentration of BamHI, the $k_{\text {off }}$ of GRDBD was found to oscillate as a function of $L$ with significant amplitude spanning a factor of 4 and a periodicity of $10 \mathrm{bp}$, which intriguingly coincides with the helical pitch of B-form DNA (Fig. 1B, red).

When we reversed the DNA sequence of the nonpalindromic GRDBD binding site (GRE) with respect to that of BamHI, the $k_{\text {off }}$ of GRDBD oscillated with a phase shift of $4 \mathrm{bp}$, nearly $180^{\circ}$ relative to that of the forward GRE (Fig. 1B). On the other hand, the binding sequence of BamHI is palindromic; therefore, its reversion is not expected to cause any phase shift.

Similar oscillatory modulation in $k_{\text {off }}$ was observed with other protein pairs, such as lac repressor (LacR) and EcoRV, or LacR and T7 RNA polymerase (T7 RNAp) (figs. S5 and S6). These proteins differ in size, shape, surface charge distribution, and DNA binding affinity (15-18). In fact, the oscillation was independent of ionic strength (fig. S7), suggesting that the electrostatic interaction between the two proteins is not the origin of the allosteric phenomenon. However, the presence of a nick, mismatched bases, or GC-rich sequences in the linker region attenuated the oscillation (figs. S8 to S10), implying that the allostery is largely dependent on the mechanical properties of the linker DNA.

To prove this hypothesis, we replaced the BamHI binding site with a DNA hairpin loop (Fig. 1C), which allows examination of the effect of DNA distortion alone. When the length of linker DNA between the hairpin loop and GRE $(L)$ was varied, we observed a similar oscillation in the $k_{\text {off }}$ of GRDBD (Fig. 1C). A larger hairpin loop decreased the amplitude of the oscillation, likely because of a smaller distortion induced by the larger hairpin (Fig. 1C). Again when GRE was reversed, the oscillation showed a 4-bp phase shift (Fig. 1D).

The oscillation dampens out with a characteristic decay length of $\sim 15$ bp (Fig. 1 and fig. S12) (12), which is much shorter than either the bending persistence length ( 150 bp) (19) or the twisting persistence length ( $\sim 300 \mathrm{bp}$ ) of DNA (20). On the other hand, recognizing that proteins primarily interact with the DNA major groove $(21,22)$, we hypothesized that allostery through DNA results mainly from distortion of the major groove.

We carried out molecular dynamics (MD) simulations first on free dsDNA in aqueous solutions at room temperature (12). We evaluated the spatial correlation between the major groove widths (R) (Fig. 2A inset) at two positions (base pairs $i$ and $i+L$ ) as a function of their separation, averaged over time $t$. We observed that the correlation coefficient has a clear oscillation with a periodicity of $\sim 10$ bp and dampens within a few helical turns (Fig. 2A). A similar yet slightly weaker oscillation was also observed for the correlation of the minor groove widths. We attribute the oscillation in Fig. 2A to thermally excited lowfrequency vibrational modes of dsDNA, which are dictated by the double helical structure of DNA.

Such spatial correlation as well as the time-averaged $R$ (Fig. 2B, red curve) are translationally invariant across a free DNA unless the symmetry is broken, as in the case of 
hairpin formation or protein binding. We simulated such an effect by applying a harmonic potential to pull a base pair apart in the middle of the strand. Under this condition, we observed that the time-averaged $R$ (Fig. 2B, blue curve) deviates from that of a free DNA (Fig. 2B, red curve) and oscillates as a function of the distance $(L)$ from the perturbed base pair with a periodicity of $\sim 10 \mathrm{bp}$. In contrast, no such oscillation was observed for the interhelical distance.

Such deviation from the free DNA, $\delta R(L)$, is expected to cause variation in the binding of the second DNA-binding protein at a distance $L$ bases away. For example, in Fig. 2C, if protein $\mathrm{B}$ widens $R$, its binding would be energetically favored at positions where $R$ is already widened $(\delta R>0)$ by the hairpin or protein A (Fig. 2C, top), but disfavored where $R$ is narrowed $(\delta R<0)$ (Fig. 2C, bottom). Consequently, reversing a nonpalindromic binding sequence would invert the binding preference of the protein, explaining the phase shift in Figure 1. This model is also well supported by the observation that the $k_{\text {off }}$ of LacR oscillates with an opposite phase in the presence of BamHI or EcoRV (fig. S6), which is consistent with the fact that BamHI widens whereas EcoRV narrows the major groove (14, $15)$.

Next, to investigate the effect of DNA allostery on transcription regulation, we studied modulation of RNA polymerase binding affinity when a protein binds near the promoter both in vitro and in vivo. The protein pair we chose is LacR and T7 RNAp, both of which, unlike GRDBD and BamHI, bend DNA $(17,23,24)$ but nevertheless exhibit a similar allosteric effect.

In the in vitro assay, we measured the binding affinity of unlabeled T7 RNAp on its promoter by titrating $k_{\text {off }}$ of labeled LacR on lac operator $\mathrm{O}_{1}$ (lacO1) with T7 RNAp. $k_{\text {off }}$ exhibited hyperbolic T7 RNAp concentration dependence (Michaelis-Menten-like kinetics) (Fig. 3, A and B, and fig. S4), as can be rigorously derived from the kinetic scheme for LacR (Protein A) and T7 RNAp (Protein B) in Fig. 3C (12):

$$
k_{\text {off }}=\frac{\left(k_{3 \rightarrow 1}+k_{3 \rightarrow 2}\right)\left(k_{1 \rightarrow 0}-k_{3 \rightarrow 2}\right)}{[\mathrm{B}] \cdot k_{1 \rightarrow 3}+\left(k_{3 \rightarrow 1}+k_{3 \rightarrow 2}\right)}+k_{3 \rightarrow 2}
$$

where $k_{i \rightarrow j}$ is the rate constant from state $i$ to $j$ and [B] is the concentration of T7 RNAp. The plateau value in the titration curve is $k_{3} \rightarrow 2$. We observed that $k_{3} \rightarrow_{2}$ oscillates as a function of $L$ with the periodicity and amplitude similar to those of GRDBD and BamHI (Fig. 3D, top).

According to Eq. 1, the dissociation constant of B on the A-bound DNA, $K_{d, B}^{\mathrm{A}}(L)$, can be measured from the value of $[\mathrm{B}]$ at which $k_{\text {off }}$ reaches half of the plateau value in the titration curves (Fig. 3, A and B) (12). We found that $K_{d, B}^{\mathrm{A}}(L)$ oscillates as a function of $L$ (Fig. 3D, middle) in phase with $k_{3 \rightarrow 2}$ [that is, $K_{d, A}^{\mathrm{B}}(L) \cdot k_{2 \rightarrow 3}$ ] (Fig. 3D, top). Therefore, the cooperativity in DNA binding, if present, exhibits either simultaneous stabilization or destabilization between the two proteins. This is a consequence of the fact that free energy is a path-independent thermodynamic state function (Fig. 3C) (12):

$$
\begin{aligned}
\Delta G_{0 \rightarrow 3}^{\circ}(L) & =\Delta G_{1 \rightarrow 3}^{\circ}(L)-\Delta G_{1 \rightarrow 0}^{\circ} \\
& =k_{B} T \ln \left(K_{d, B}^{\mathrm{A}}(L) \cdot K_{d, A}^{\circ}\right) \\
& \left.=\Delta G_{2 \rightarrow 3}^{\circ}(L)-\Delta G_{2 \rightarrow 0}^{\circ}\right) \\
& =k_{B} T \ln \left(K_{d, A}^{\mathrm{B}}(L) \cdot K_{d, B}\right)
\end{aligned}
$$


where $K_{\mathrm{d}, \mathrm{A}}$ and $K_{\mathrm{d}, \mathrm{B}}$ are the dissociation constant of a protein in the absence of the other, $k_{\mathrm{B}}$ is the Boltzmann constant, and $\mathrm{T}$ is temperature.

Based on the second line of Eq. 2, the free energy of the ternary complex, $\Delta G_{0 \rightarrow 3}^{\circ}(L)$, was found to oscillate with a periodicity of $\sim 10 \mathrm{bp}$ and an amplitude of $\sim 2 k_{B} T$ (Fig. 3D, bottom).

In general, for a ternary complex formed with DNA and Proteins A and B, the free energy of the overall system is $\Delta G_{0 \rightarrow 3}^{\circ}(L)=\Delta G_{\mathrm{A}}^{\circ}+\Delta G_{\mathrm{B}}^{\circ}+\Delta \Delta G_{\mathrm{AB}}^{\circ}(L)$, where $\Delta G_{\mathrm{A}}^{\circ}$ and $\Delta G_{\mathrm{B}}^{\circ}$ are the binding free energies of the two individual proteins on DNA, respectively. $\Delta \Delta G_{\mathrm{AB}}^{\circ}$, small relative to $\Delta G_{\mathrm{A}}^{\circ}$ or $\Delta G_{\mathrm{B}}^{\circ}$, is the energetic coupling involving in the linker DNA, given by the sum of two terms - the variation of protein A binding caused by protein $B$ and the variation of protein $B$ binding caused by protein A:

$$
\Delta \Delta G_{\mathrm{AB}}^{\circ}(L) \infty \delta R_{\mathrm{A}}^{\mathrm{A}} \delta R_{\mathrm{A}}^{\mathrm{B}}(L)+\delta R_{B}^{A}(L) \delta R_{\mathrm{B}}^{\mathrm{B}}
$$

In each $\delta R$, or the distortion of the major groove widths, the subscripts indicate where the distortion occurs (binding site of protein $\mathrm{A}$ or $\mathrm{B}$ ) and the superscripts indicate the protein that causes the DNA distortion (12). According to our proposed mechanism, $\delta R_{\mathrm{A}}^{\mathrm{B}}(L)$ and $\delta R_{\mathrm{B}}^{\mathrm{A}}(L)$ propagate periodically (Fig. $2 \mathrm{~B}$ ), yielding a damped oscillation in $\Delta \Delta G_{\mathrm{AB}}^{\circ}(L)$. This explains the oscillations of the coupling energy for LacR and T7 RNAp (Fig. 3D, bottom and fig. S14A) and for GRDBD and BamHI (fig. S14B).

The allosteric coupling between LacR and T7 RNAp is likely to affect transcription in vivo because the efficiency of transcription initiation correlates with the binding affinity of T7 RNAp (25). We therefore inserted DNA templates used in vitro (fig. S15) into the chromosome of Escherichia coli and measured the expression level of lacZ using the Miller assay (Fig. 4A) (26). Indeed, the gene expression level oscillates as a function of $L$ with a periodicity of $\sim 10$ bp (Fig. 4B). Similar oscillations of T7 RNAp activity were observed on plasmids in $E$. coli cells by using a yellow fluorescent protein as a reporter (fig. S16). The oscillation of gene expression levels with a 10-bp periodicity was also seen in a classic experiment on lac operon with a DNA loop formed by two operators (27). However, our T7 RNAp result illustrates that DNA allostery results in such an oscillatory phenomenon even without a DNA loop, which is consistent with a recent study in which E. coli RNA polymerase was used (10).

Pertinent to eukaryotic gene expression, DNA allostery may affect the binding affinity of transcription factors near nucleosomes that are closely positioned $(28,29)$. We placed GRE downstream of a nucleosome (Fig. 4C) and observed a similar DNA allosteric effect in the $k_{\text {off }}$ of GRDBD (Fig. 4D, and fig. S17). To evaluate DNA allostery in an internucleosomal space, we used two nucleosomes to flank a GRE (Fig. 4C). At the same separation $L$, GRDBD resides on GRE for a relatively longer time with a single nucleosome nearby than it does with a pair of nucleosomes on both sides of GRE (Fig. 4D). Nonetheless, the fold change between the maximal and minimal $k_{\text {off }}$ is larger for GRDBD with two nucleosomes (approximately sevenfold). This indicates moderately large cooperativity between the two flanking nucleosomes in modifying the binding affinity of GRDBD, which is in line with previous in vivo experiments $(30,31)$. The fact that histones modify a neighboring transcription factor's binding suggests that allostery through DNA might be physiologically important in affecting gene regulation. 


\section{Supplementary Material}

Refer to Web version on PubMed Central for supplementary material.

\section{Acknowledgments}

We thank K. Wood for his early involvement and J. Hynes, A. Szabo, C. Bustamante and J. Gelles for helpful discussions. This work is supported by NIH Director's Pioneer Award and NIH grant GM096450 to X.S.X., Peking University for BIOPIC, Thousand Youth Talents Program for Y.S., as well as the Major State Basic Research Development Program (2011CB809100), National Natural Science Foundation of China (31170710, 31271423, 21125311).

\section{References and Notes}

1. Monod J, Wyman J, Changeux JP. J. Mol. Biol. 1965; 12:88. [PubMed: 14343300]

2. Koshland DE, Némethy G, Filmer D. Biochemistry. 1966; 5:365. [PubMed: 5938952]

3. Pohl FM, Jovin TM, Baehr W, Holbrook JJ. Proc. Natl. Acad. Sci. U.S.A. 1972; 69:3805. [PubMed: 4509343]

4. Hogan M, Dattagupta N, Crothers DM. Nature. 1979; 278:521. [PubMed: 372825]

5. Parekh BS, Hatfield GW. Proc. Natl. Acad. Sci. U.S.A. 1996; 93:1173. [PubMed: 8577735]

6. Rudnick J, Bruinsma R. Biophys. J. 1999; 76:1725. [PubMed: 10096873]

7. Panne D, Maniatis T, Harrison SC. Cell. 2007; 129:1111. [PubMed: 17574024]

8. Moretti R, et al. ACS Chem. Biol. 2008; 3:220. [PubMed: 18422304]

9. Koslover EF, Spakowitz AJ. Phys. Rev. Lett. 2009; 102:178102. [PubMed: 19518837]

10. Garcia, Hernan G., et al. Cell Reports. 2012; 2:150. [PubMed: 22840405]

11. Kim S, Blainey PC, Schroeder CM, Xie XS. Nat. Methods. 2007; 4:397. [PubMed: 17435763]

12. Materials and methods are available as supplementary materials on Science online

13. Luisi BF, et al. Nature. 1991; 352:497. [PubMed: 1865905]

14. Newman M, Strzelecka T, Dorner L, Schildkraut I, Aggarwal A. Science. 1995; 269:656.

[PubMed: 7624794]

15. Winkler FK, et al. EMBO J. 1993; 12:1781. [PubMed: 8491171]

16. Lewis M, et al. Science. 1996; 271:1247. [PubMed: 8638105]

17. Kalodimos CG, et al. EMBO J. 2002; 21:2866. [PubMed: 12065400]

18. Durniak KJ, Bailey S, Steitz TA. Science. 2008; 322:553. [PubMed: 18948533]

19. Smith S, Finzi L, Bustamante C. Science. 1992; 258:1122. [PubMed: 1439819]

20. Bryant Z, et al. Nature. 2003; 424:338. [PubMed: 12867987]

21. Travers AA. Annu. Rev. Biochem. 1989; 58:427. [PubMed: 2673015]

22. Rohs R, et al. Annu. Rev. Biochem. 2010; 79:233. [PubMed: 20334529]

23. Újvári A, Martin CT. J. Mol. Biol. 2000; 295:1173. [PubMed: 10653695]

24. Tang G-Q, Patel SS. Biochemistry. 2006; 45:4936. [PubMed: 16605261]

25. Jia Y, Kumar A, Patel SS. J. Biol. Chem. 1996; 271:30451. [PubMed: 8940010]

26. Miller, JH. Experiments in molecular genetics. Cold Spring Harbor Laboratory; 1972.

27. Müller J, Oehler S, Müller-Hill B. J. Mol. Biol. 1996; 257:21. [PubMed: 8632456]

28. Lee W, et al. Nat. Genet. 2007; 39:1235. [PubMed: 17873876]

29. Sharon E, et al. Nat. Biotech. 2012; 30:521.

30. John S, et al. Nat. Genet. 2011; 43:264. [PubMed: 21258342]

31. Meijsing SH, et al. Science. 2009; 324:407. [PubMed: 19372434]

32. Viadiu H, Aggarwal AK. Nat. Struct. Biol. 1998; 5:910. [PubMed: 9783752]

33. Daber R, Stayrook S, Rosenberg A, Lewis M. J. Mol. Biol. 2007; 370:609. [PubMed: 17543986]

34. Lowary PT, Widom J. J. Mol. Biol. 1998; 276:19. [PubMed: 9514715]

35. Strähle U, Klock G, Schütz G. Proc. Natl. Acad. Sci. U.S.A. 1987; 84:7871. [PubMed: 2891134]

Science. Author manuscript; available in PMC 2013 August 15. 
36. Tang G-Q, Bandwar RP, Patel SS. J. Biol. Chem. 2005; 280:40707. [PubMed: 16215231]

37. Joo, C.; Ha, T. Single molecule techniques: a laboratory manual. Selvin, PR.; Ha, T., editors. Cold Spring Harbor Laboratory Press; Cold Spring Harbor: 2008. p. 3-35.

38. Eismann ER, Müller-Hill B. J. Mol. Biol. 1990; 213:763. [PubMed: 2359123]

39. Elf J, Li G-W, Xie XS. Science. 2007; 316:1191. [PubMed: 17525339]

40. Chen J, Matthews KS. J. Biol. Chem. 1992; 267:13843. [PubMed: 1629185]

41. Gottesfeld JM, et al. J. Mol. Biol. 2001; 309:615. [PubMed: 11397084]

42. Schroeder, CM.; Blainey, PC.; Kim, S.; Xie, XS. Single molecule techniques: a laboratory manual. Selvin, PR.; Ha, T., editors. Cold Spring Harbor Laboratory Press; Cold Spring Harbor: 2008. p. 461-492.

43. Blainey PC, van Oijen AM, Banerjee A, Verdine GL, Xie XS. Proc. Natl. Acad. Sci. U.S.A. 2006; 103:5752. [PubMed: 16585517]

44. Luo G, Wang M, Konigsberg WH, Xie XS. Proc. Natl. Acad. Sci. U.S.A. 2007; 104:12610. [PubMed: 17640918]

45. Gilbert W, Müller-Hill B. Proc. Natl. Acad. Sci. U.S.A. 1966; 56:1891. [PubMed: 16591435]

46. Datsenko KA, Wanner BL. Proc. Natl. Acad. Sci. U.S.A. 2000; 97:6640. [PubMed: 10829079]

47. Lee EC, et al. Genomics. 2001; 73:56. [PubMed: 11352566]

48. Oehler S, Eismann ER, Krämer H, Müller-Hill B. EMBO J. 1990; 9:973. [PubMed: 2182324]

49. Munteanu MG, Vlahovicek K, Parthasarathy S, Simon I, Pongor S. Trends Biochem. Sci. 1998; 23:341. [PubMed: 9787640]

50. Emsley P, Cowtan K. Acta Crystallogr. D. 2004; 60:2126. [PubMed: 15572765]

51. Cheetham GMT, Jeruzalmi D, Steitz TA. Nature. 1999; 399:80. [PubMed: 10331394]

52. Dolinsky TJ, Nielsen JE, McCammon JA, Baker NA. Nucl. Acids Res. 2004; 32:W665. [PubMed: 15215472]

53. Li H, Robertson AD, Jensen JH. Proteins. 2005; 61:704. [PubMed: 16231289]

54. Baker NA, Sept D, Joseph S, Holst MJ, McCammon JA. Proc. Natl. Acad. Sci. U.S.A. 2001; 98:10037. [PubMed: 11517324]

55. Case, D., et al. AMBER 11. University of California; San Francisco: 2010.

56. Berendsen HJC, Grigera JR, Straatsma TP. J. Phys. Chem. 1987; 91:6269. 1987/11/01.

57. Pérez A, et al. Biophys. J. 2007; 92:3817. [PubMed: 17351000]

58. Ryckaert J-P, Ciccotti G, Berendsen HJC. J. Comput. Phys. 1977; 23:327.

59. Darden T, York D, Pedersen L. J. Chem. Phys. 1993; 98:10089.

60. Berendsen HJC, Postma JPM, Gunsteren W. F. v. DiNola A, Haak JR. J. Chem. Phys. 1984; $81: 3684$.

61. Falcon CM, Matthews KS. Biochemistry. 2000; 39:11074. [PubMed: 10998245]

62. Romanuka J, et al. J. Mol. Biol. 2009; 390:478. [PubMed: 19450607]

63. Perona JJ. Methods. 2002; 28:353. [PubMed: 12431439]

64. Vipond IB, Baldwin GS, Halford SE. Biochemistry. 1995; 34:697. [PubMed: 7819265]

65. Horton NC, Perona JJ. Proc. Natl. Acad. Sci. U.S.A. 2000; 97:5729. [PubMed: 10801972]

66. Israelachvili, J. Intermolecular \& Surface Forces. 2nd. Academic Press; 1991.

67. Baumann CG, Smith SB, Bloomfield VA, Bustamante C. Proc. Natl. Acad. Sci. U.S.A. 1997; 94:6185. [PubMed: 9177192]

68. Wenner JR, Williams MC, Rouzina I, Bloomfield VA. Biophys. J. 2002; 82:3160. [PubMed: 12023240]

69. Gore J, et al. Nature. 2006; 439:100. [PubMed: 16397501]

70. Hormeño S, et al. Biophys. J. 2011; 100:1996. [PubMed: 21504736]

71. Yin YW, Steitz TA. Science. 2002; 298:1387. [PubMed: 12242451]

72. Holmes VF, Cozzarelli NR. Proc. Natl. Acad. Sci. U.S.A. 2000; 97:1322. [PubMed: 10677457]

73. Dillon SC, Dorman CJ. Nat. Rev. Microbiol. 2010; 8:185. [PubMed: 20140026]

74. Jain A, et al. Nature. 2011; 473:484. [PubMed: 21614075] 
75. Vasudevan D, Chua EYD, Davey CA. J. Mol. Biol. 2010; 403:1. [PubMed: 20800598] 
A

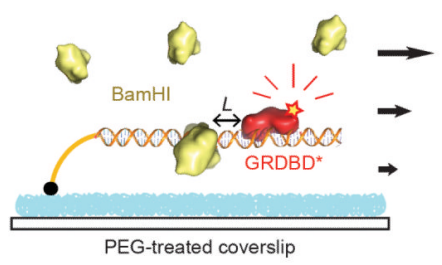

C

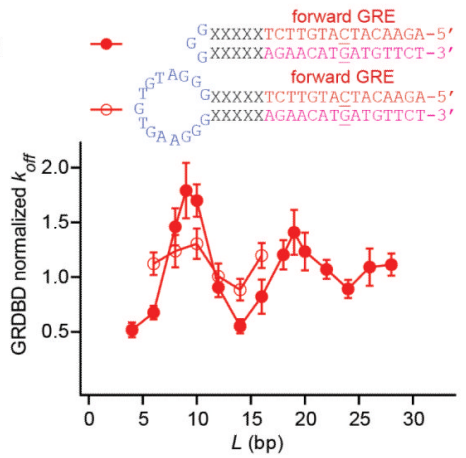

B

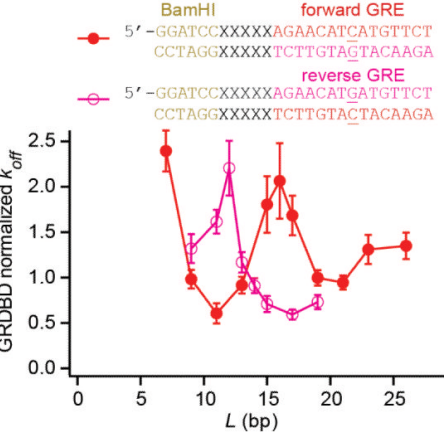

D
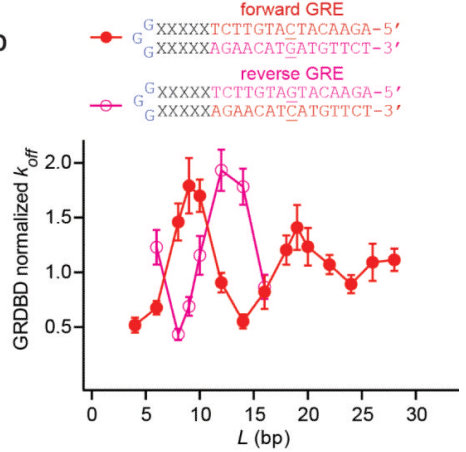

Fig. 1.

Allostery through DNA affecting $k_{\text {off }}$ of GRDBD near BamHI or near a hairpin loop. (A) Schematic for the single-molecule assay in a flow cell. The structural model is for $L=11$ with GRDBD from Protein Data Bank (PDB) ID 1R4R (13) and BamHI from PDB ID 2BAM (32). (B) Oscillation in the $k_{\text {off }}$ of GRDBD for the forward (red solid circles) and reverse (magenta open circles) GRE sequences, normalized to that measured in the absence of BamHI $( \pm$ SEM). DNA sequences are shown with the linker DNA $(L=5)$. The central base of GRE, which makes the sequence non-palindromic, is underlined. (C) Protein binding affinity affected by a nearby DNA hairpin loop, 3 bp and 15 bp $( \pm$ SEM). (D) Effect of 3-bp loop on the forward and reverse GRE $( \pm \mathrm{SEM})$. The DNA sequence is shown for $L=5 . k_{\text {off }}$ is normalized to that measured on DNA without a hairpin loop. 


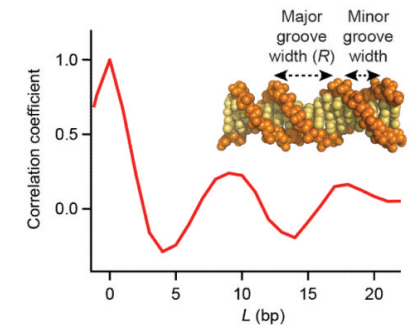

B

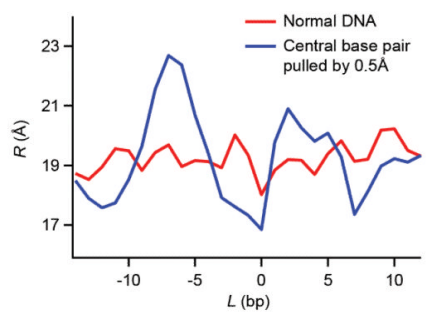

C

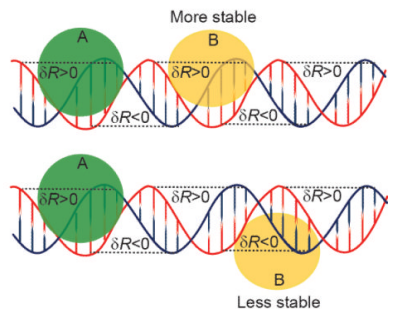

Fig. 2.

Allostery through DNA induced by distortion of the major groove. (A) MD simulation at room temperature reveals the spatial correlation between the major groove widths (inset, defined as the distance between $\mathrm{C} 3$ atoms of the $i^{\text {th }}$ and $i+7^{\text {th }}$ nucleotide sugar-rings) at two positions as a function of their separation $L$, averaged over time $t,\left[\langle\delta R(i ; t) \delta R(i+L ; t)\rangle_{t}\right.$, $i=5]$. The correlation oscillates with a periodicity of $\sim 10 \mathrm{bp}$ and is attributed to thermally excited low-frequency vibrational modes of dsDNA. (B) Upon breaking the symmetry by pulling apart a base pair in the middle of the dsDNA (defined as $L=0$ ) by $0.5 \AA$ (12), the time-averaged $R$ (blue) deviates from that of a free DNA (red) and oscillates as a function of the distance $(L)$ from the perturbed base pair with a periodicity of $\sim 10 \mathrm{bp}$. (C) Oscillation of $R(L)$ causes the variation of the allosteric coupling between two DNA-binding proteins A and B. If protein B widens $R$, it would energetically favor binding at positions where $R$ is already widened $(\delta R>0)$ by protein A (top), but disfavor where $R$ is narrowed $(\delta R<0)$ (bottom). 
A

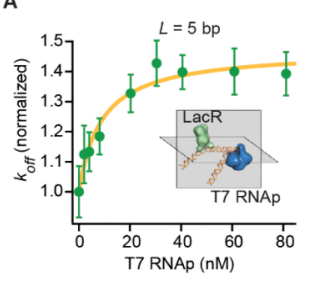

c

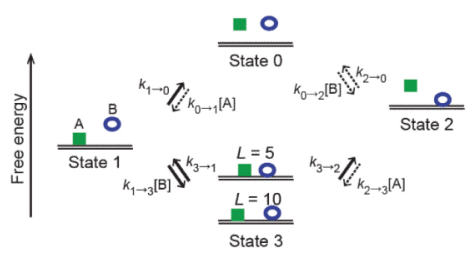

D
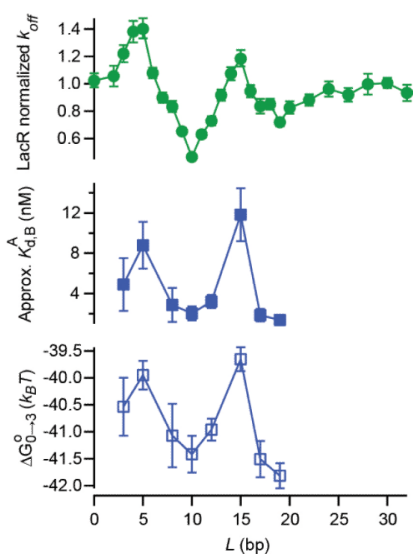

Fig. 3.

Allostery through DNA between LacR and T7 RNAp in vitro. (A and B) Titration curves, where $k_{\text {off }}$ values were normalized to those measured in the absence of T7 RNAp on the given template $( \pm$ SEM). The hyperbolic fit (yellow) is based on Eq. 1. Structural models illustrate the ternary complex of LacR [PDB ID 2PE5 (33)] and T7 RNAp [PDB ID 3E2E (18)]. (C) Kinetic model for the binding of proteins A (LacR) and B (T7 RNAp). Our experiments start with state 1 and proceed to the dissociation of LacR to state 0 or state 2 (via state 3), as shown with solid arrows. Dashed arrows indicate reactions that are not considered in our derivation of Eq.1. (D) The maximum $k_{\text {off }}$ of LacR $\left(k_{3} \rightarrow 2\right), K_{\mathrm{d}}$ of T7

RNAp in the presence of $\operatorname{LacR}\left(K_{d, B}^{\mathrm{A}}\right)$, and the free energy of the ternary complex $\left(\Delta G_{0 \rightarrow 3}^{\circ}\right)$, as function of $L$, oscillating with a periodicity of $10 \mathrm{bp}$. Error bars reflect SEM for $k_{3} \rightarrow 2$ and $1 \mathrm{SD}$ of the $\chi^{2}$ fit for $K_{d, B}^{\mathrm{A}}$ and $\Delta G_{0 \rightarrow 3}^{\circ}(12)$. 
A BgllI

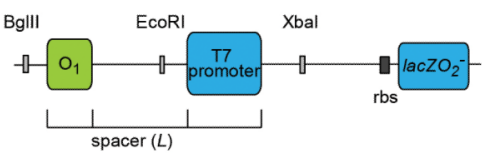

B

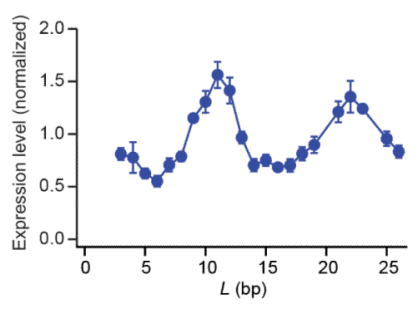

c

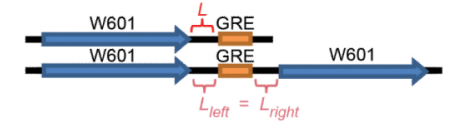

D

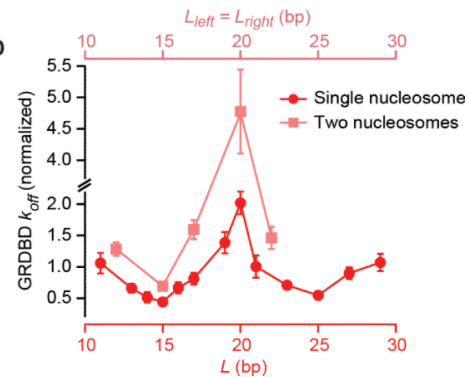

Fig. 4.

Physiological relevance of DNA allostery. (A) E. coli strains constructed to examine cooperativity between LacR and T7 RNAp on the bacterial chromosome. (B) The expression level of lac $Z$ (normalized to the average expression levels of all $L$ s) oscillates as a function of $L$ with a periodicity of $10 \mathrm{bp}$, similar to the corresponding in vitro data (fig. S15). Error bars reflect SEM $(n=3)$. (C) Schematic for the DNA sequences used in the GRDBDnucleosome experiment. W601 is the Widom 601 nucleosome positioning sequence (34). (D) Oscillation of the $k_{\text {off }}$ of GRDBD as a function of $L( \pm \mathrm{SEM})$. Data was normalized to $k_{\text {off }}$ of GRDBD in the absence of histone (fig. S17). 